\title{
L'éducation, priorité du système scolaire russe
}

\section{Dominique Thoirain}

\section{OpenEdition}

\section{Journals}

Édition électronique

URL : http://journals.openedition.org/ries/4174

DOI : $10.4000 /$ ries.4174

ISSN : 2261-4265

\section{Éditeur}

Centre international d'études pédagogiques

\section{Édition imprimée}

Date de publication : 5 mars 1995

Pagination : 133-141

ISSN : 1254-4590

\section{Référence électronique}

Dominique Thoirain, «L'éducation, priorité du système scolaire russe », Revue internationale d'éducation de Sèvres [En ligne], 05 | 1995, mis en ligne le 16 mars 2015, consulté le 02 mai 2019. URL : http:// journals.openedition.org/ries/4174; DOI : 10.4000/ries.4174

Ce document a été généré automatiquement le 2 mai 2019.

(c) Tous droits réservés 


\title{
L'éducation, priorité du système scolaire russe
}

\author{
Dominique Thoirain
}

1 L'école russe, comme hier l'école soviétique, fonde sa mission sur un projet éducatif. Dans les deux systèmes d'enseignement, éduquer (vospitat) représente une fonction aussi importante qu'instruire (obrasovat). Elle se dilue dans l'ensemble de l'activité scolaire mais ne fait pas l'objet, en elle-même, d'une discipline distincte.

2 La loi-cadre de juillet 1992 sur l'enseignement ne dicte pas explicitement de valeurs, à la différence des lois-cadre sur l'éducation de l'époque soviétique. Elle fait allusion aux «valeurs universelles ", à l'épanouissement de l'individu et elle insiste sur la fonction éducative de l'école. C'est un point commun fondamental entre la nouvelle école russe et l'école soviétique. Deux valeurs sont seulement explicitées dans la loi de 1992 : le sens du civisme et l'amour de la patrie. La seconde était une valeur centrale dans l'école soviétique ${ }^{1}$.

3 Ces remarques montrent d'emblée que l'école d'aujourd'hui s'inscrit dans une continuité avec l'ancienne, bien que des valeurs humanistes ou religieuses, inexistantes dans le projet éducatif soviétique, soient maintenant prônées.

4 Nous ne pouvons comprendre ni le tâtonnement présent dans la mise en place d'un nouveau projet éducatif, ni la virulence avec laquelle on oppose de nouvelles valeurs aux anciennes sans avoir effectué un retour sur l'ancien système éducatif, ses valeurs et les méthodes utilisées pour les inculquer.

\section{Les valeurs dans l'école soviétique}

5 Ce que nous entendons ici par "valeur » s'appuie sur les termes utilisés par Raymond Boudon et François Bourricaud ${ }^{2}$ pour caractériser ce concept :

Dans toute société, la détermination des objectifs s'effectue à partir d'une représentation du désirable et se manifeste dans les idéaux collectifs. Ces valeurs qui, systématiquement ordonnées, s'organisent en une vision du monde, 
apparaissent souvent comme une donnée irréductible, un noyau stable, un ensemble de variables indépendantes.

6 Au cours des soixante-dix années de l'histoire mouvementée de l'Union soviétique, le projet éducatif a connu des modifications dans son contenu. « Le code moral du bâtisseur du communisme ", adopté en 1961 par le congrès du Parti communiste de l'URSS, codifiait les valeurs du système éducatif, telles qu'elles ont été inculquées jusqu'aux années quatre-vingt. Elles tendaient à atteindre quatre objectifs :

- former des citoyens politiquement actifs et instruits,

- encourager l'amour de la patrie et la solidarité internationale prolétarienne,

- développer le désir de défendre la patrie,

- prôner l'athéisme.

7 Dans ce texte de 1961, il n'est pas fait mention d'une autre valeur, le collectif, conçue comme un moyen et comme une fin dans le projet éducatif soviétique, ainsi que le formulait Anton Makarenko: «éduquer dans le collectif, par le collectif et pour le collectif $»$.

8 Comment ces valeurs étaient-elles inculquées aux élèves ? L'organisation de la vie scolaire facilitait la tâche, grâce à la continuité de lieu et de temps qui caractérisait le déroulement de la scolarité.

9 La continuité de lieu: l'enfant entrait à 7 ans dans une école (à 6 ans à partir de la réforme de 1984) et n'en sortait qu'à 17 ans, s'il y avait accompli les dix années d'enseignement général (onze années à partir de 1984). Il fréquentait ainsi pendant dix années les mêmes lieux, le même personnel d'encadrement et les mêmes professeurs avec, cependant, un changement de type d'enseignants à la charnière du cycle secondaire, à partir de la sixième année. La continuité dans le temps : après les cinq ou six heures quotidiennes de cours, la plupart des élèves restaient à l'école et y participaient le reste de l'après-midi à des activités périscolaires (clubs de divers types, activités sportives, organisation de fêtes...).

10 L'action éducative s'inscrivait aussi dans la continuité grâce à l'encadrement pédagogique qui inculquait les valeurs, que ce soient les enseignants, en classe, ou les animateurs, pendant les activités périscolaires. Les enseignants étaient tous obligatoirement membres $\mathrm{du}$ syndicat unique des personnels d'éducation et les plus méritants parmi eux appartenaient au parti communiste. Les animateurs provenaient tous du personnel d'encadrement des mouvements de jeunesse, c'est-à-dire du mouvement des Pionniers ou du mouvement des Jeunesses communistes (le Komsomol). Ceci explique le monolithisme ou la cohésion - du discours éthique de ces pédagogues qui avaient intériorisé leur fonction sociale et savaient qu'ils devaient transmettre les valeurs qui leur avaient été explicitement énoncées.

11 Quant à l'élève, son statut était clairement défini lorsqu'il commençait sa scolarité. Il n'était pas un élève quelconque d'une quelconque division scolaire : il était pionnier. Arborant solennellement, dès le jour de la rentrée, un uniforme (chemise blanche et foulard rouge avec une jupe unie pour les filles, ou un pantalon pour les garçons), il prêtait le serment de membre de l'organisation des Pionniers. À 14 ans, c'est par une autre cérémonie qu'il intégrait le mouvement du Komsomol. Cette pratique rituelle avait une fonction éducative évidente : faire que le jeune fasse siennes les valeurs prônées. Or, il était attribué une fonction d'organisation de la vie scolaire à ces deux mouvements. Ils prenaient en charge, en fonctionnant en brigades, une grande partie de l'entretien des 
locaux ou bien assuraient la responsabilité de l'ordre et de la discipline dans les couloirs de l'école au moment des interclasses. Donc, dès l'âge de sept ans, l'enfant se sentait responsabilisé au sein d'une communauté qui allait lui inculquer des valeurs.

L'organisation rituelle de la vie scolaire atteignait son point d'orgue lors des fêtes de l'école. Celle du début et celle de la fin de l'année scolaire, la fête de la "première cloche " et celle de la "dernière cloche ", étaient marquées par un épisode qui exprimait le lien unissant les différentes cohortes d'élèves: la plus jeune élève de l'école, perchée sur les épaules d'un grand garçon d'une classe terminale, faisait le tour de la cour d'école où se tenaient pour la circonstance les élèves, les enseignants et les invités d'honneur, en faisant carillonner la cloche, image du début ou de la fin de l'année scolaire. Nul doute que ce moment impressionnaient les jeunes enfants qui commençaient leurs vies d'élèves.

D'autres fêtes célébraient une valeur particulière. Nous pensons aux fêtes scolaires consacrées au patriotisme. Plusieurs journées lui étaient consacrées dans le calendrier soviétique : le 23 février, journée du soldat et du garde-frontière et le 9 mai, journée commémorative de la victoire remportée contre l'occupant nazi.

Nous avons assisté, en 1984, à l'école $\mathrm{n}^{\circ} 105$ de Kharkov, au déroulement de ces deux fêtes. Elles réunissaient pour l'après-midi et la soirée, outre les différents usagers de l'école, des personnalités représentant la municipalité, l'administration régionale de l'éducation, les entreprises recevant les élèves pour les "activités pratiques » et l'école d'officiers du district de la ville où se situait l'école. Les anciens combattants de la deuxième guerre mondiale, arborant leurs médailles honorifiques, étaient aussi présents. Plusieurs générations se trouvaient alors mélangées et l'occasion était donnée aux plus âgés d'évoquer leur passé et de faire connaître leur expérience de la guerre aux jeunes pour qui la défense de la patrie était un concept abstrait. La première partie de l'aprèsmidi était consacrée aux discours. Puis, un concours interclasses réunissait les élèves des trois dernières années, vêtus de treillis. Il s'agissait, d'une part, de jeux martiaux - monter et démonter un fusil, tirer à l'arc - et, d'autre part, de compétitions sportives. Le jury se composait d'anciens combattants et d'officiers. Ainsi, il était demandé aux élèves de démontrer leur aptitude physique à défendre le pays. En fin de soirée, on livrait l'école aux élèves qui en transformaient le préau en salle de bal.

15 Nous décelons, dans le déroulement de ces deux fêtes, le souci du personnel d'encadrement d'adapter les cérémonies rituelles du patriotisme au goût des jeunes afin qu'elles ne deviennent pas rébarbatives. La fête trouvait ici, dans le cadre scolaire, sa fonction éducative : tisser des liens entre générations et faciliter la transmission d'une valeur.

16 Mais si la fête était un moment privilégiée pour la formation éthique, c'était par le contenu des cours qu'elle s'effectuait, chaque jour. En nous intéressant plus particulièrement à l'enseignement de l'histoire ${ }^{3}$, nous nous attacherons, ici encore, à discerner les mécanismes d'inculcation de la valeur patriotique. L'analyse de plusieurs pages de manuels scolaires ${ }^{4}$, montre comment les auteurs impliquent le lecteur, c'est-àdire l'élève, dans des événements qui ont marqué l'histoire de son pays. Ceci concerne les chapitres consacrés aux guerres.

Par exemple, la guerre de 1812 qui rejeta Napoléon hors du sol russe est communément appelée dans les manuels soviétiques : «la première guerre patriotique ». Évoquant la bataille de Borodino, un auteur écrit: "Elle apparaît comme exemplaire du combat héroïque de l'armée et du peuple russes pour l'indépendance du pays ». Ainsi, à partir 
d'une victoire de l'armée impériale, au début du XIX siècle, cet ouvrage cherchait à forger le sentiment patriotique d'un jeune du troisième tiers du $\mathrm{XX}^{\mathrm{e}}$ siècle.

La deuxième guerre mondiale est communément nommée: "la grande guerre patriotique ». Une impression domine à la lecture des chapitres qui lui sont consacrés : c'est avant tout grâce au front soviétique et à l'Armée rouge que la guerre a tourné à l'avantage des Alliés. Les auteurs du manuel étudié ne prennent aucun recul pour évoquer les faits: le front soviétique, c'est "nous", les troupes hitlériennes, ce sont "nos" ennemis. Et l'on favorise ainsi chez l'élève la formation d'une attitude manichéenne qui différencie les «bons » des «méchants ». On perçoit ici une faiblesse de cette formation éducative fondée sur un discours pédagogique dont le contenu est pauvre, parce qu'il privilégie le récit d'épisodes militaires au détriment de l'analyse, et qui est peu adapté à des élèves de quinze ou seize ans, aptes à réfléchir et à caractériser des systèmes politiques sans qu'on leur indique préalablement ceux qu'ils doivent condamner. Notons aussi que ce type de discours véhicule implicitement une valeur qui dépasse le patriotisme et qui s'apparente plutôt au nationalisme en affirmant, ici, la supériorité militaire du peuple soviétique ou, ailleurs, celle de la nation russe.

C'est cette discipline, l'histoire, qui a, avec la littérature, le plus subi la censure et cela pendant des dizaines d'années. Les manuels occultaient des faits nombreux de l'histoire soviétique, faits qui constituent ce que l'on appelle depuis la Perestroika: «les pages blanches » des manuels. C'est aussi depuis ce tournant que l'écriture de l'histoire est devenue l'objet privilégié du travail des journalistes dans leur quête de transparence (glasnost) : la contestation du discours historique de la période soviétique a été si intense que l'épreuve écrite d'histoire a disparu de l'examen final des études secondaires.

Malgré ses faiblesses, cet enseignement a laissé des traces dans la conscience patriotique et la culture des jeunes. Nous l'avons perçu dans les résultats d'enquêtes menées par des sociologues soviétiques à la fin des années quatre-vingt ou par ceux de notre propre travail de terrain, conduit à la fin de l'année 1991, dans deux grandes villes: Moscou et Kharkov. L'utilisation des phrases toutes faites, tout droit sorties du manuel, ou des stéréotypes que celui-ci utilisait prouvait que la mémorisation avait contribué à donner forme au patriotisme.

21 Le discours explicite n'était pas le seul moyen d'inculquer des valeurs. Le contenu de l'énoncé d'un problème de mathématiques, d'une leçon de lecture ou d'un exercice de grammaire offraient de fréquentes opportunités pour inculquer une valeur de manière implicite.

22 En feuilletant un manuel de grammaire russe de cinquième année, réédité en 1990, nous avons relevé différents exemples développant le thème patriotique. Divers exercices évoquant la Constitution de l'URSS, la décoration de l'Ordre de Lénine, la commémoration du 9 mai, les qualités requises pour être un citoyen exemplaire, le sens de l'honneur et du dévouement des Pionniers...

En ne mobilisant pas la partie consciente de l'activité intellectuelle de l'apprenant, centrée sur l'exercice, mais son inconscient qui n'en enregistrait pas moins le message, la démarche éducative devenait perverse.

Évoquons, enfin, une dernière discipline qui contribuait à former le jeune patriote : la formation militaire. La formation militaire ne concernait que les élèves des deux dernières années de l'école secondaire, à raison de deux heures hebdomadaires. Elle était réservée aux garçons, les filles recevant une formation de secouriste. Elle consistait en un 
apprentissage du maniement des armes avec, en fin d'année scolaire, un stage de cinq jours en caserne. La formation était assurée par un officier rattaché à l'école où il animait aussi certaines activités périscolaires ou certaines fêtes, comme nous l'avons vu précédemment. Cette formation, perçue comme désuète par les élèves, visait plutôt à entretenir dans la conscience des jeunes l'idée d'une possible menace extérieure, à laquelle ils devraient savoir parer si elle devenait une réalité.

Ainsi, dans le système scolaire soviétique, la fonction éducative s'effectuait grâce à un ensemble cohérent de pratiques fondé sur un énoncé explicite de valeurs. Dans cette machinerie qui paraissait bien huilée, le contenu, les valeurs, n'étaient pas séparables du mode d'inculcation, l'organisation de la vie scolaire. C'est cet ensemble qui s'est peu à peu désintégré, par étapes, sans que l'idée d'un projet éducatif scolaire soit abandonné et avec l'apparition tâtonnante de nouvelles valeurs.

\section{Les valeurs de la nouvelle école russe}

Ce sont les activités périscolaires qui ont été touchées en premier par la vague de transformations. À partir de 1987, les mouvements de Pionniers et du Komsomol ont perdu leur monopole d'organisateurs et de cadres de la jeunesse: divers mouvements sont apparus, certains à caractère religieux, d'autres politiques, écologiques... La Loi sur l'éducation de 1992 interdisant toute activité sociopolitique à l'intérieur des écoles et des universités (article 50, § 15), les premiers ont été éliminés de la vie scolaire et, avec eux, ce sont les activités périscolaires qui ont perdu leurs cadres. Elles s'organisent maintenant hors de l'école mais sous la forme d'associations à but lucratif, le plus souvent. Le déroulement de la vie scolaire doit être repensé en l'absence des brigades qui organisaient l'autodiscipline parmi les élèves.

La disparition de la formation militaire a été plus tardive. La loi de 1992 lui retire son caractère obligatoire. Elle ne peut être dispensée qu'avec le consentement des parents et des élèves eux-mêmes et le coût de cette formation ne relève plus de l'école mais de l'institution militaire qui propose cette formation. Avec la dégradation très prononcée de l'image de l'armée parmi les jeunes et le souhait très souvent formulé par ceux-ci, dans les enquêtes, de ne pas accomplir leur service militaire, cette formation ne concerne plus qu'une petite minorité de jeunes.

28 Le discours éthique de l'école a été transformé lorsque le système éducatif soviétique a implosé avec la disparition du monopole d'État en matière d'éducation qui n'a d'ailleurs pas été brutale. Elle a commencé avec l'autorisation accordée par l'État aux institutions privées, russes ou autres, de subventionner des écoles publiques ou de fonder leurs propres écoles. Cette pratique, reconnue par la loi-cadre de 1992, est apparue en 1989 : des écoles privées ont été créées, d'abord sous forme d'établissements fournissant aux familles des répétiteurs ou bien d'« écoles à la maison». Un an plus tôt, en 1988, des " écoles du dimanche ", confessionnelles, s'étaient ouvertes à Moscou : c'était le premier pas vers la création d'écoles religieuses, nombreuses aujourd'hui dans les grandes villes.

Mais la fin du monopole d'État n'est pas tellement due à l'apparition des écoles privées. Leur effectif total reste faible : on en dénombre, en 1994, 368 sur le territoire de la Russie qui compte au total 64700 établissements d'enseignements de niveau primaire et secondaire, soit $0,5 \%$ de l'ensemble; et leur existence parait souvent éphémère : elles ne fonctionnent qu'une ou deux années, le plus souvent. 

1992 qui a fait voler en éclats le monopole d'état. Leurs objectifs éducatifs sont extrêmement divers : certaines se donnent pour objectif de rechercher et de former des enfants surdoués - c'est le cas du Fonds Soros, d'origine américaine, d'autres proposent leurs services aux écoles pour former des jeunes au management. Et il y a les institutions religieuses, l'Église orthodoxe ou bien la Fondation internationale biblique, pour ne citer que les plus connues, qui se proposent de prendre en charge la formation religieuse des élèves.

31 Un nouveau discours éducatif a remplacé l'ancien. Il se compose de valeurs opposées aux anciennes et il varie selon le lieu de scolarisation de l'enfant. De ce fait, les familles choisissent l'école que fréquentera leur enfant en fonction de leur propre projet éducatif. Notons qu'à la diversité des écoles publiques ou privées correspond aussi une hiérarchie de coûts car, à l'exception d'un fond minimal d'enseignements obligatoires, la formation est payante et varie selon le prestige des disciplines et des enseignants.

Il est possible, cependant, de dégager une orientation éthique commune à l'ensemble des écoles publiques. Elle caractérise le projet éducatif de l'école russe de cette première moitié des années quatre-vingt-dix. Elle émane principalement de l'État russe lui-même, et des institutions religieuses, l'Église orthodoxe et, accessoirement des missions d'origine anglo-saxonne qui propagent le contenu de la Bible.

Nous évoquerons trois éléments de cette orientation: la priorité que l'école doit accorder à l'épanouissement de l'individu, la nécessité de donner aux élèves une formation religieuse et la formation patriotique.

loi-cadre de 1992 proclame dans son article 2 que « ... la politique de l'État en matière d'éducation repose sur... le développement libre de la personne... ».

Ceci s'entend comme une déclaration condamnant le projet soviétique qui se fondait sur le collectif. On peut trouver l'origine de cette affirmation dans le manifeste publié dès 1986 par un groupe de pédagogues, les «novateurs ", déjà renommés par leurs travaux et leurs expériences pédagogiques. Ce groupe a affirmé son opposition aux dogmes de l'Académie des sciences pédagogiques et développé ses propositions éducatives centrées sur le respect de la personne de l'enfant et du jeune, de leur libre épanouissement. Son projet s'est fondé sur l'expérience et les écrits de pédagogues étrangers, principalement John Dewey et Maria Montessori qui affirment comme premier principe pédagogique, le respect du rythme de développement de chaque enfant.

Ce n'est pas par un texte législatif que l'instruction religieuse est entrée à l'école. Elle s'est peu à peu imposée au rythme des transformations que la vie publique russe a connues depuis 1987 et qui ont donné une place de plus en plus importante aux autorités ecclésiastiques orthodoxes. C'est à l'automne 1989 que la presse a rapporté pour la première fois l'existence de cours d'histoire religieuse dans certaines écoles publiques de certaines grandes villes d'URSS. Cela coïncidait avec le renouveau religieux, la multiplication des baptêmes et l'afflux de participants aux offices.

Selon John Dustan ${ }^{5}$, ce renouveau religieux a pour fonction « de régénérer moralement la société. Le monde communiste éprouvait de la difficulté à subsister alors que les mots avaient perdu leur sens et que les gens n'avaient plus confiance en lui. Un vide avait été créé. La morale religieuse, a-t-on dit, était le seul moyen possible de combler le vide ». En 1990, la Loi sur la liberté de conscience affirmait que l'éducation athéiste était bannie de

Revue internationale d'éducation de Sèvres, 05 | 2015 
l'école mais, auparavant, l'année précédente, une autre loi avait réaffirmé la séparation de l'Église et de l'État.

Pour les responsables des instituts pédagogiques, l'enseignement religieux est perçu comme un chapitre de l'enseignement de l'histoire qui avait été jusqu'ici négligé. Ils attendent la collaboration des institutions religieuses qui, seules, peuvent apporter leur savoir à l'école russe, héritière de l'école soviétique et donc ignorante dans ce domaine.

Des recueils de textes religieux, des extraits de la Bible sont édités pour les maîtres dans les éditions pédagogiques avec le concours des instances orthodoxes ou des missions étrangères bibliques. L'attitude des enseignants face à cette nouvelle discipline est variable : elle va de l'acquiescement au rejet. Cette deuxième attitude se comprend quand on se souvient que ces mêmes enseignants devaient, voici moins de dix ans, inculquer l'athéisme à leurs élèves. Le refus qu'expriment certains d'entre eux d'aborder le thème religieux avec leurs élèves peut provenir soit d'un sentiment d'incompétence, soit d'une conviction philosophique que les transformations politiques récentes n'ont pas émoussée.

L'épiscopat orthodoxe ne conçoit pas, quant à lui, cet enseignement religieux comme une banale discipline scolaire. Il s'agit, pour lui, d'une formation qui doit permettre à chaque jeune « d'épanouir en lui ce qu'il y a de plus précieux et qui lui a été donné à sa naissance ». Ces ecclésiastiques affirment aussi la nécessité de l'enseignement religieux pour former le citoyen russe : «La religion orthodoxe est un élément identitaire du Russe au même titre que la langue russe ». Ainsi, la foi orthodoxe devient un élément constitutif du nouveau contenu donné au sentiment patriotique.

41 Mais la patrie a changé d'échelle avec la disparition de l'URSS (1991) et c'est sans doute la redéfinition du contenu de cette valeur qui cause le plus grand nombre de tâtonnements. La détermination de la nation russe s'effectue difficilement car l'éclatement fut brutal et l'histoire de l'empire fait que la Russie peut être conçue comme un espace s'étendant bien au-delà des limites de l'ex-République fédérative de Russie. De nombreuses familles ont des parents résidant dans les autres républiques de l'ex-URSS.

42 La patrie a aussi changé d'envergure internationale et il est sans doute douloureux et difficile, pour les éducateurs d'aujourd'hui, d'évoquer cette dégradation. À moins que la nostalgie de la puissance ne l'emporte et colore de nationalisme l'éducation patriotique. Nous avions vu précédemment que cette tendance nationaliste pointait déjà dans le discours scolaire soviétique.

43 Au terme de cette évocation du projet éducatif russe, rappelons qu'il a repris à son compte le souci d'éduquer qu'avait affirmé l'école soviétique et c'est un cas original au regard des écoles européennes occidentales.

Nous pensons, par ailleurs, que ce projet russe s'appauvrit lorsqu'il se définit en opposition au système soviétique et qu'il s'élabore sur le rejet d'un héritage pédagogique riche malgré ses contradictions. Il pratique ainsi l'amnésie, l'irresponsabilité face au passé, ce qui risque de pervertir les relations entre les différentes générations d'enseignants et entre enseignants et enseignés. 


\section{NOTES}

1. Dominique Thoirain, Valeurs, école, jeunesse : le cas du patriotisme dans l'école soviétique, thèse de doctorat soutenue en juillet 1993, à Paris-VIII.

2. Raymond Boudon, François Bourricaud, Dictionnaire critique de la sociologie, PUF, Paris, 1986.

3. L'auteur de l'article enseigne cette discipline en France.

4. II n'y a qu'un seul manuel, rarement deux, par discipline et par niveau. Chacun était édité à plusieurs millions d'exemplaires.

5. John Dustan, Soviet education and perestroika, Rootledge, New York, 1992.

6. Ces propos sont extraits du compte rendu d'une rencontre entre des directeurs d'instituts pédagogiques de l'enseignement public et les membres de l'épiscopat orthodoxe, paru dans Le journal de l'enseignant, $\mathrm{n}^{\circ} 32$ de 1994.

\section{RÉSUMÉS}

Le système scolaire russe connaît de profonds bouleversements, étroitement liés aux transformations politiques qui ont provoqué l'éclatement de l'unité idéologique et géographique de l'URSS. L'émergence de valeurs nouvelles, totalement opposées à celles de l'Ancien Régime, caractérise la législation actuelle sur l'école. Mais les deux systèmes présentent néanmoins un aspect commun essentiel : la volonté de conférer une mission d'« éducation » à l'enseignement.

\section{INDEX}

Index géographique : Russie

Mots-clés : éducation morale, éthique, système éducatif, valeur, éducation

\section{AUTEUR}

\section{DOMINIQUE THOIRAIN}

Professeur de géographie, docteur en sciences de l'éducation, France 